\title{
The Strange Case of Privacy in Equilibrium Models
}

\author{
RACHEL CUMMINGS, California Institute of Technology \\ KATRINA LIGETT, Hebrew University and California Institute of Technology \\ MALLESH M. PAI, University of Pennsylvania \\ AARON ROTH, University of Pennsylvania
}

We study how privacy technologies affect user and advertiser behavior in a simple economic model of targeted advertising. In our model, a consumer first decides whether or not to buy a good, and then an advertiser chooses an advertisement to show the consumer. The consumer's value for the good is correlated with her type, which determines which ad the advertiser would prefer to show to her-and hence, the advertiser would like to use information about the consumer's purchase decision to target the ad that he shows.

In our model, the advertiser is given only a differentially private signal about the consumer's behaviorwhich can range from no signal at all to a perfect signal, as we vary the differential privacy parameter. This allows us to study equilibrium behavior as a function of the level of privacy provided to the consumer. We show that this behavior can be highly counter-intuitive, and that the effect of adding privacy in equilibrium can be completely different from what we would expect if we ignored equilibrium incentives. Specifically, we show that increasing the level of privacy can actually increase the amount of information about the consumer's type contained in the signal the advertiser receives, lead to decreased utility for the consumer, and increased profit for the advertiser, and that generally these quantities can be non-monotonic and even discontinuous in the privacy level of the signal.

A full version of this paper is available online at http://arxiv.org/abs/1508.03080

The first author was supported in part by a Simons Award for Graduate Students in Theoretical Computer Science, NSF grant CNS-1254169, and US-Israel Binational Science Foundation grant 2012348. The second author was supported in part by NSF grants CNS-1254169 and CNS-1518941, DARPA-BAA-15-29, US-Israel Binational Science Foundation grant 2012348, the Charles Lee Powell Foundation, a Google Faculty Research Award, an Okawa Foundation Research Grant, a Microsoft Faculty Fellowship, a subcontract through the DARPA Brandeis project, a grant from the HUJI Cyber Security Research Center, and a startup grant from Hebrew University's School of Computer Science. The third author was supported in part by NSF Grant CCF-1101389. The fourth author was supported in part by NSF Grant CCF-1101389, an NSF CAREER award, DARPA-BAA-15-29, and an Alfred P. Sloan Foundation Fellowship. Part of this work was completed during a stay at the Simons Institute for the Theory of Computing at Berkeley.

Authors' addresses: R. Cummings, Computing and Mathematical Sciences, California Institute of Technology. email: rachelc@caltech.edu; K. Ligett, School of Computer Science and Engineering, Hebrew University, and Department of Computing and Mathematical Sciences and Division of the Humanities and Social Sciences, California Institute of Technology. email: katrina@caltech.edu; M.M. Pai, Department of Economics, University of Pennsylvania. email: mallesh@econ.upenn.edu; A. Roth, Department of Computer and Information Science, University of Pennsylvania. email: aaroth@cis.upenn.edu.

Permission to make digital or hard copies of part or all of this work for personal or classroom use is granted without fee provided that copies are not made or distributed for profit or commercial advantage, and that copies bear this notice and the full citation on the first page. Copyrights for third-party components of this work must be honored. For all other uses, contact the owner/author(s). Copyright is held by the author/owner(s).

EC'16, July 24-28, 2016, Maastricht, The Netherlands.

ACM 978-1-4503-3936-0/16/07.

http://dx.doi.org/10.1145/2940716.2940740 